\title{
Adrenomedullin refines mortality prediction by the BODE index in COPD: the "BODE-A" index
}

\author{
Daiana Stolz, Kostantinos Kostikas, Francesco Blasi, Wim Boersma, \\ Branislava Milenkovic, Alicia Lacoma, Renaud Louis, Joachim G. Aerts, \\ Tobias Welte, Antoni Torres, Gernot G.U. Rohde, Lucas Boeck, Janko Rakic, \\ Andreas Scherr, Sabine Hertel, Sven Giersdorf and Michael Tamm
}

Affiliations: For the authors' affiliations see the Acknowledgements section.

Correspondence: D. Stolz, Clinic of Pneumology and Pulmonary Cell Research, University Hospital Basel, Petersgraben 4, CH-4031 Basel, Switzerland. E-mail: daiana.stolzQusb.ch

ABSTRACT The BODE (body mass index, airflow obstruction, dyspnoea, exercise capacity) index is wellvalidated for mortality prediction in chronic obstructive pulmonary disease (COPD). Concentrations of plasma pro-adrenomedullin, a surrogate for mature adrenomedullin, independently predicted 2-year mortality among inpatients with COPD exacerbation.

We compared accuracy of initial pro-adrenomedullin level, BODE and BODE components, alone or combined, in predicting 1-year or 2-year all-cause mortality in a multicentre, multinational observational cohort with stable, moderate to very severe COPD.

Pro-adrenomedullin was significantly associated $(\mathrm{p}<0.001)$ with 1 -year mortality $(4.7 \%)$ and 2-year mortality $(7.8 \%)$ and comparably predictive to BODE regarding both (C statistics 0.691 versus 0.745 and 0.635 versus 0.679 , respectively). Relative to using BODE alone, adding pro-adrenomedullin significantly improved 1-year and 2-year mortality prognostication (C statistics 0.750 and 0.818 , respectively; both $\mathrm{p}<0.001$ ). Pro-adrenomedullin plus BOD was more predictive than the original BODE including 6-min walk distance. In multivariable analysis, pro-adrenomedullin (likelihood ratio Chi-squared 13.0, p $<0.001$ ), body mass index $(8.5, \mathrm{p}=0.004)$ and 6 -min walk distance $(7.5, \mathrm{p}=0.006)$ independently foretold 2-year survival, but modified Medical Research Council dyspnoea score $(2.2, p=0.14)$ and forced expiratory volume in $1 \mathrm{~s} \%$ predicted $(0.3, \mathrm{p}=0.60)$ did not.

Pro-adrenomedullin plus BODE better predicts mortality in COPD patients than does BODE alone; proadrenomedullin may substitute for 6-min walk distance in BODE when 6-min walk testing is unavailable.

-

@ERSpublications

Pro-adrenomedullin improves BODE prediction of mortality in COPD patients and may substitute for 6-min walk distance http://ow.ly/qV5M3

This article has supplementary material available from www.erj.ersjournals.com

For editorial comments see page 322 .

Received: April 022013 | Accepted after revision: June 052013 | First published online: June 212013

Clinical trial: This study is registered at www.controlled-trials.com with identifier number ISRCTN99586989.

Support statement: This work was supported by the Pulmonary Medicine Clinic, University Hospital Basel, Basel, Switzerland and by the Swiss National Foundation (grant PP00-P3_128412/1). Thermo Scientific Biomarkers (formerly Brahms AG), Hennigsdorf, Germany, manufacturer of the relevant assays, provided pro-adrenomedullin, pro-calcitonin, copeptin and pro-atrial natriuretic peptide reagents gratis, and, through an unrestricted research grant, defrayed blood sample transportation to the central study laboratory at University Hospital Basel and also supported the services of an English-language medical editor.

Conflict of interest: Disclosures can be found alongside the online version of this article at www.erj.ersjournals.com Copyright @ERS 2014 


\section{Introduction}

A major goal of assessing patients with chronic obstructive pulmonary disease (COPD) is determining risk of adverse outcomes including mortality, so as to guide interventions $[1,2]$. Initially, forced expiratory volume in $1 \mathrm{~s}$ (FEV1) was the main factor used in mortality prognostication in COPD, but increasing recognition of the predictive power of systemic and perceptive variables prompted development of multidimensional risk assessment tools $[2,3]$. One well-validated multidimensional tool is the BODE (body mass, airflow obstruction, dyspnoea, exercise capacity) index, determined based on the body mass index, FEV1 \% predicted, modified Medical Research Council (mMRC) dyspnoea grade, and 6-min walk distance (6MWD) [2]. However, BODE may incompletely capture the impact of the systemic manifestations and comorbidities of COPD; indeed, the tool's originators recognised the latter limitation by introducing a comorbidity index described as complementing BODE [4]. Furthermore, time, space, staffing and equipment requirements make BODE's 6-min walk test (6MWT) component cumbersome in primary care or other busy or resource-constrained settings, driving development of alternative or additional multidimensional tools $[5,6]$.

Therefore, interest has mounted in applying systemic biomarkers to adverse outcome prediction in COPD patients [7-9]. One candidate is plasma pro-adrenomedullin, the stable, apparently biologically-inactive midregional fragment of the adrenomedullin prohormone [10, 11]. Adrenomedullin is a pluripotent regulatory peptide acting as both a hormone and a cytokine to exert extensive vascular, immunomodulatory and metabolic effects $[11,12]$. The finding of circulating adrenomedullin elevation in end-stage lung disease [13] prompted a prospective, single-centre observational cohort study of pro-adrenomedullin in adverse outcome prediction in inpatients with COPD exacerbation [14]; in multivariate analysis incorporating 12 demographic, clinical, spirometric and laboratory factors, this study found that admission proadrenomedullin concentration was the only independent predictor of 2-year all-cause mortality [14].

This observation led us to hypothesise that pro-adrenomedullin might have similar predictive ability for 1and 2-year all-cause mortality in patients with clinically stable COPD. We also hypothesised that proadrenomedullin might provide additional prognostic information to that offered by BODE and BODE components. We therefore envisioned that combining pro-adrenomedullin and BODE might improve longterm mortality prediction compared to use of the latter alone. Furthermore, we speculated that proadrenomedullin plus the non-6MWT BODE components ("BOD”) might have comparable prognostic accuracy to that of the original BODE including 6MWD.

Therefore, we conducted the present prospectively planned analysis with the primary aim of evaluating the accuracy of a single baseline circulating pro-adrenomedullin estimation, alone or combined with BODE or BOD, to predict 1-year or 2-year all-cause mortality in a multinational, multicentre, prospective, longitudinal, observational cohort study of patients with clinically stable COPD.

\section{Methods}

Study overview

Designed to be inclusive, exploratory and hypothesis-generating, the Predicting Outcome using Systemic Markers in Severe Exacerbations of Chronic Obstructive Pulmonary Disease (PROMISE-COPD) Study evaluated variables potentially identifying poor outcomes during and outside exacerbations in patients with moderate to very severe COPD, i.e. post-bronchodilator FEV1/forced vital capacity $<70 \%$ and $\mathrm{FEV} 1<80 \%$ pred or Global Initiative for Chronic Obstructive Lung Disease grade II-IV airway obstruction. Enrollees had a baseline examination, and then were followed for $\geqslant 2$ years in scheduled semi-annual visits. Additionally, as necessary, patients made outpatient visits or were hospitalised for treatment of COPD exacerbation; follow-up visits were specified for 4 weeks post-exacerbation onset. Throughout the study, patients were treated as clinically warranted, without restriction.

PROMISE-COPD was approved by the participating centres' ethics committees, and registered at www. controlled-trials.com (identifier ISRCTN99586989). Patients provided prior written informed consent for all study assessments.

\section{Patients}

638 patients were consecutively recruited and followed at 11 European hospital pneumology departments from November 2008 to October 2011. Inclusion criteria comprised: 1) at baseline, clinically stable, moderate to very severe COPD based on anamnesis, physical examination and spirometry $\geqslant 4$ weeks after the latest exacerbation resolved; 2) age $\geqslant 40$ years; and 3) smoking history $\geqslant 10$ pack-years. Exclusion criteria were: 1) main respiratory disorder other than COPD; 2) death expected within 6 months; 


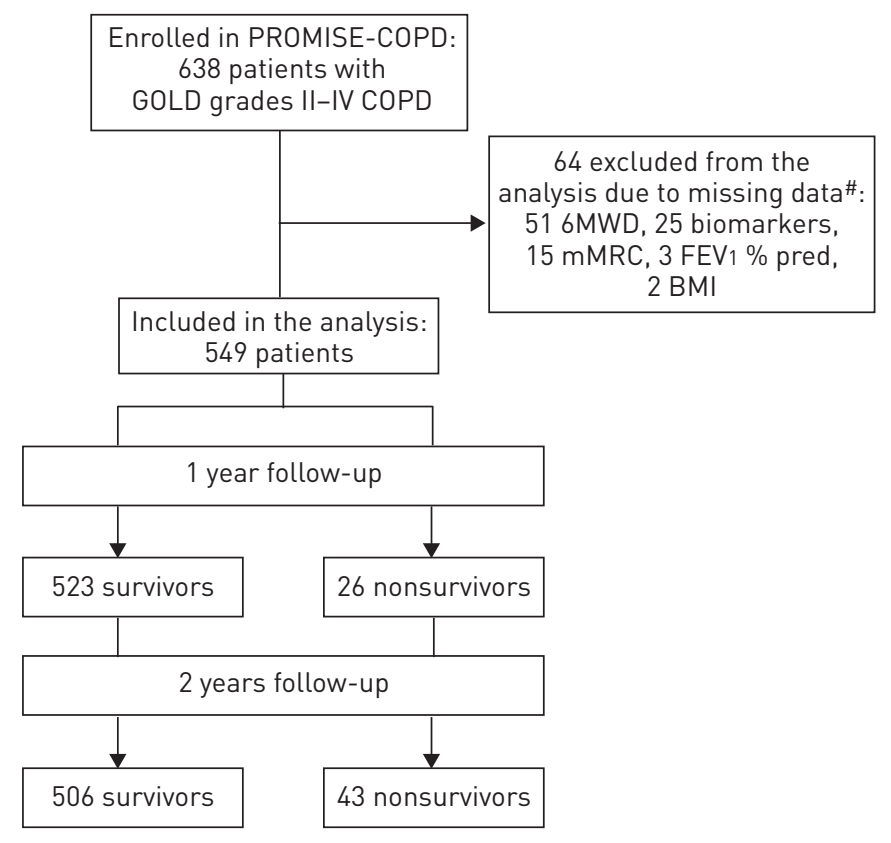

FIGURE 1 Flow chart for the present analysis. Missing data, which led to exclusion from the present analysis, were as follows: pro-adrenomedullin $(n=24)$, procalcitonin $(n=24)$, copeptin $(n=24)$, pro-atrial natriuretic peptide $(n=25)$, body mass index (BMI) $(\mathrm{n}=2)$, forced expiratory volume in $1 \mathrm{~s}(\mathrm{FEV} 1)(\mathrm{n}=3)$, modified Medical Research Council (mMRC) dyspnoea score $(n=15)$ and 6-min walk distance $(6 M W D)(n=51)$. Of the 43 nonsurvivors, 26 died during the first year of follow-up, 17 during the second year. PROMISE-COPD: Predicting Outcome using Systemic Markers in Severe Exacerbations of Chronic Obstructive Pulmonary Disease Study; GOLD: Global Initiative for Chronic Obstructive Lung Disease; COPD: chronic obstructive pulmonary disease; $\%$ pred: $\%$ predicted. ${ }^{\#}$ : some patients were missing data for more than one variable.

3) immunosuppression, including AIDS, organ transplantation or chronic steroids $\left(>20 \mathrm{mg} \cdot \mathrm{day}^{-1}\right.$ prednisolone equivalent); and 4) musculoskeletal or neuromuscular disorder preventing walking.

\section{Baseline assessment}

For each patient, we performed a physical examination, registered vital signs and obtained a detailed history. Spirometry and 6MWTs were administered by trained technicians following American Thoracic Society guidelines $[15,16]$. Patients completed the mMRC dyspnoea questionnaire in local languages, in validated versions when available. BODE scoring employed original methods [2].

\section{Biomarkers}

Besides pro-adrenomedullin, PROMISE-COPD evaluated several plasma biomarkers (online supplementary material). Biomarker quantification was performed in duplicate within one run in a central, accredited laboratory by technicians unaware of patients' clinical data, using automated sandwich immunoassays (Kryptor; Thermo Scientific Biomarkers, Hennigsdorf, Germany). The pro-adrenomedullin assay had a 0.05-100 nmol $\cdot \mathrm{L}^{-1}$ measurement range and $0.25 \mathrm{nmol} \cdot \mathrm{L}^{-1}$ functional sensitivity. The pro-adrenomedullin assay has been shown to exhibit low intra-assay variability, and concentrations of pro-adrenomedullin appear stable in samples that are stored at room temperature for up to $72 \mathrm{~h}$, undergo multiple freeze-thaw cycles or are drawn from patients in a nonfasting state [11].

The reference interval of pro-adrenomedullin in a healthy subset is $0.23-0.64 \mathrm{nmol} \cdot \mathrm{L}^{-1}$ (median $0.41 \mathrm{nmol} \cdot \mathrm{L}^{-1}$ ). Age, measures of obesity (body mass index and body fat percentage), blood lipids (cholesterol, low-density lipoprotein and triglycerides), glucose tolerance (haemoglobin A1c), smoking, alcohol, inflammation (C-reactive protein), renal function (cystatin $\mathrm{C}$ ) and cardiac wall stress (N-terminal pro-brain natriuretic peptide) were significantly associated with circulating pro-adrenomedullin levels [17].

\section{Outcome assessment}

1-year and 2-year vital status, the outcomes of interest in this analysis, were confirmed at the time of scheduled study visits. If needed for this purpose, we contacted relatives, family physicians or insurers, and checked medical records, hospital databases and public registries, to determine dates and causes of deaths. 
TABLE 1 Baseline characteristics for the analysed cohort and according to 2-year survival

\begin{tabular}{|c|c|c|c|c|}
\hline & Analysed cohort & 2-year survivors & 2-year nonsurvivors & p-value ${ }^{\#}$ \\
\hline Subjects $\mathrm{n}$ & 549 & 506 & 43 & \\
\hline Age years & $66.0 \pm 11.4$ & $65.8 \pm 11.6$ & $69.1 \pm 8.7$ & 0.066 \\
\hline Male & $69.8(383)$ & $70.6(357)$ & $60.5(26)$ & 0.167 \\
\hline Caucasian & 99.1 (544) & $99.2(502)$ & $97.7(42)$ & 0.158 \\
\hline Current smoker & $33.3(183)$ & $33.0(167)$ & $37.2(16)$ & 0.714 \\
\hline Pack-years & $45.0 \pm 31.1$ & $45.0 \pm 31.0$ & $50.0 \pm 21.2$ & $<0.001$ \\
\hline Body mass index $\mathrm{kg} \cdot \mathrm{m}^{-2}$ & $26.1 \pm 5.4$ & $26.2 \pm 5.3$ & $24.5 \pm 5.3$ & 0.051 \\
\hline mMRC dyspnoea score & $3(2-3)$ & $2(2-3)$ & $3(2-4)$ & 0.001 \\
\hline 6-min walk distance m & $390 \pm 131$ & $380 \pm 108$ & $305 \pm 109$ & $<0.001$ \\
\hline BODE score & $3(1-4)$ & $3(1-4)$ & $4(2-7)$ & $<0.001$ \\
\hline BOD score ${ }^{+}$ & $2(1-4)$ & $2(1-4)$ & $3(2-5)$ & $<0.001$ \\
\hline \multicolumn{5}{|l|}{ Comorbidities } \\
\hline Arterial hypertension & $51.4(282)$ & $51.0(258)$ & $55.8(24)$ & 0.663 \\
\hline Coronary arterial disease & $23.7(130)$ & $22.9(116)$ & $32.6(14)$ & 0.192 \\
\hline Congestive heart failure & $14.4(79)$ & $14.2(72)$ & $16.3(7)$ & 0.896 \\
\hline Myocardial infarction & $9.3(51)$ & $9.9(50)$ & $2.3(1)$ & 0.170 \\
\hline Pulmonary hypertension & $9.7(53)$ & $9.3(47)$ & $14.0(6)$ & 0.477 \\
\hline Malignancy & $3.8(21)$ & $4.0(20)$ & $2.3(1)$ & 0.851 \\
\hline Diabetes mellitus & $12.0(66)$ & $11.5(58)$ & $18.6(8)$ & 0.317 \\
\hline Renal failure & $6.0(33)$ & $5.7(29)$ & $9.3(4)$ & 0.177 \\
\hline Adjusted Charlson score & $4(3-5)$ & $4(3-5)$ & $4(3-6)$ & 0.038 \\
\hline \multicolumn{5}{|l|}{ GOLD grade } \\
\hline II & $50.8(279)$ & 51.2 (259) & $46.5(20)$ & 0.738 \\
\hline III & $34.1(187)$ & $34.2(173)$ & $32.6(14)$ & \\
\hline IV & $15.1(83)$ & $14.6(74)$ & $20.9(9)$ & \\
\hline Post-bronchodilator FVC \% pred & $78.7 \pm 23.9$ & $77.8 \pm 24.2$ & $78.7 \pm 20.5$ & 0.994 \\
\hline Post-bronchodilator FEV $1 \%$ pred & $48.9 \pm 18.3$ & $49.4 \pm 18.5$ & $45.9 \pm 16.8$ & 0.234 \\
\hline Post-bronchodilator FEV1/FVC \% & $48.3 \pm 14.1$ & $48.6+14.2$ & $44.7 \pm 13.1$ & 0.084 \\
\hline Pro-adrenomedullin nmol. $\mathrm{L}^{-1}$ & $0.60(0.48-0.79)$ & $0.59(0.48-0.78)$ & $0.78(0.51-1.20)$ & 0.006 \\
\hline Procalcitonin $\mu \mathrm{g} \cdot \mathrm{L}^{-1}$ & $0.08(0.07-0.1)$ & $0.08(0.07-0.10)$ & $0.09(0.07-0.11)$ & 0.019 \\
\hline Copeptin pmol $\cdot \mathrm{L}^{-1}$ & $8.8(2.4-15.2)$ & $8.6(2.4-14.7)$ & $10.9(5.5-28.9)$ & 0.033 \\
\hline Pro-atrial natriuretic peptide $\mathrm{pmol} \cdot \mathrm{L}^{-1}$ & $82.0(52.4-140.4)$ & $80.8(51.9-136.0)$ & $109.9(60.4-205.7)$ & 0.018 \\
\hline
\end{tabular}

Data are presented as mean \pm SD, \% (n) or median (interquartile range), unless otherwise stated. mMRC: modified Medical Research Council; BODE: body mass, airflow obstruction, dyspnoea, exercise capacity index; GOLD: Global Initiative for Chronic Obstructive Lung Disease; FVC: forced vital capacity; \% pred: \% predicted; FEV1: forced expiratory volume in $1 \mathrm{~s} .{ }^{\#}$ : 2-year survivors versus 2-year nonsurvivors. ${ }^{\circ}$ : scores range, in increasing severity, from 0 to $4 .^{+}$: BOD comprises BODE without the exercise capacity measurement; the three BOD components are scored according to the same cut-offs as they are in BODE [2], therefore, the "BOD" index ranges, in increasing severity, from 0 to 7.

\section{Statistics}

Continuous variables are expressed as mean $\pm \mathrm{SD}$ or median (interquartile range (IQR)), and discrete variables as $\mathrm{n}(\%)$ or vice versa. Baseline characteristics were compared between survivors and nonsurvivors using the Mann-Whitney U-test or the t-test.

To establish the relationships with 1-year or 2-year all-cause mortality of pro-adrenomedullin or BODE variables, alone or combined, we created univariate and multivariable Cox proportional hazard regression models. Biomarker values were log-transformed to conform to the models' linearity assumption between predictor and outcome [18]. Proportional hazard assumptions were tested for all modelled variables; results are given as hazard ratios with 95\% confidence intervals and significance levels for Chi-squared (Wald) test. Hazard ratios were standardised to describe a one-quartile change in each analysed variable.

The C statistic, bootstrap-corrected for multivariable models, is given as a measure of accuracy in classifying patients regarding survival. The $\mathrm{C}$ statistic is equivalent to the area under the receiver-operating characteristics curve. Larger values denote greater accuracy: the null value is 0.5 , the maximum 1.0.

Incremental discrimination from adding blood biomarkers to BODE or BOD is shown using the likelihood ratio Chi-squared test for nested models [18] and the net reclassification improvement [19], which summarises the correct change in risk categories (downwards for survivors and upwards for nonsurvivors) with a tested predictive method versus the reference method. All analyses utilised a two-sided $p=0.05$ for significance. 
TABLE 2 Cox regression models to predict 1-year and 2-year all-cause mortality

HR $(95 \% \mathrm{CI})$

p-value

C statistic

Model

Chi-squared

\begin{tabular}{|c|c|c|c|c|c|}
\hline \multicolumn{6}{|c|}{ 1-year all-cause mortality } \\
\hline \multicolumn{6}{|l|}{ Univariate } \\
\hline BODE & $3.30(2.04-5.35)$ & $<0.001$ & 0.745 & 23.24 & $<0.001$ \\
\hline $\mathrm{BOD}^{\#}$ & $3.74(1.90-7.34)$ & $<0.001$ & 0.690 & 15.25 & $<0.001$ \\
\hline Pro-adrenomedullin & $2.36(1.67-3.35)$ & $<0.001$ & 0.691 & 19.28 & $<0.001$ \\
\hline \multicolumn{6}{|l|}{ Multivariate } \\
\hline BODE & $3.18(1.91-5.28)$ & $<0.001$ & & & \\
\hline BODE plus pro-adrenomedullin & $2.18(1.53-3.09)$ & $<0.001$ & 0.818 & 39.56 & $<0.001$ \\
\hline $\mathrm{BOD}^{\#}$ & $3.84(1.92-7.66)$ & $<0.001$ & & & \\
\hline BOD $^{\#}$ plus pro-adrenomedullin & $2.35(1.65-3.34)$ & $<0.001$ & 0.800 & 34.38 & $<0.001$ \\
\hline \multicolumn{6}{|l|}{ 2-year all-cause mortality } \\
\hline \multicolumn{6}{|l|}{ Univariate } \\
\hline BODE & $2.53(1.73-3.69)$ & $<0.001$ & 0.679 & 22.07 & $<0.001$ \\
\hline $\mathrm{BOD}^{\#}$ & $3.74(1.90-7.34)$ & $<0.001$ & 0.649 & 15.26 & $<0.001$ \\
\hline Pro-adrenomedullin & $1.95(1.45-2.62)$ & $<0.001$ & 0.635 & 16.73 & $<0.001$ \\
\hline \multicolumn{6}{|l|}{ Multivariate } \\
\hline BODE & $2.42(1.64-3.57)$ & $<0.001$ & & & \\
\hline BODE plus pro-adrenomedullin & $1.80(1.34-2.42)$ & 0.001 & 0.750 & 35.72 & $<0.001$ \\
\hline $\mathrm{BOD}^{\#}$ & $2.81(1.66-4.78)$ & 0.001 & & & \\
\hline $\mathrm{BOD}^{\#}$ plus pro-adrenomedullin & $1.91(1.42-2.59)$ & $<0.001$ & 0.738 & 31.48 & $<0.001$ \\
\hline
\end{tabular}

For each variable, the hazard ratio (HR) is based on an increment of one-quartile. $p$-values compare variable with $\mathrm{HR}=1$ or $\mathrm{C}$ statistics $=0.5$ (null hypothesis). BODE: body mass, airflow obstruction, dyspnoea, exercise capacity index. \#: BOD comprises BODE without the exercise capacity measurement; the three BOD components are scored according to the same cut-offs as they are in BODE [2], therefore, the "BOD index" ranges, in increasing severity, from 0 to 7 .

\section{Results}

$549(86.1 \%)$ of 638 PROMISE-COPD patients had complete biomarker and BODE variable data and were included in the analysis (fig. 1 and table 1). Enrollees excluded for missing biomarker measurements $(n=25)$ did not differ from analysed patients in sex, race or BODE component values or score (data not shown).

Median (IQR) follow-up for the 549 analysed patients was 722 (395-762) days. After 2 years, 43 (7.8\%) patients had expired; 26 (4.7\%; 60.5\% of deaths) during the first year and $17(3.1 \%$; $39.5 \%$ of deaths) during the second year. The commonest causes of mortality were respiratory insufficiency attributed to COPD $(34.9 \%)$ and cardiovascular events $(18.6 \%)$.

Table 1 compares baseline characteristics of 2-year survivors versus 2-year nonsurvivors. Collectively, these subgroups had similar demographics and airflow limitation, but nonsurvivors had higher BODE scores, a greater burden of comorbidities (reflected by the age-adjusted Charlson score) and significantly higher levels of pro-adrenomedullin and the other plasma biomarkers studied. Pro-adrenomedullin levels at all four stable visits increased over time $(\mathrm{p}=0.01)$ and with nonsurvivor status $(\mathrm{p}=0.0006)$. However, there was no difference between the slope of increase in survivors and nonsurvivors over time ( $\mathrm{p}=0.516$, mixed-effect model).

Table 2 presents results of Cox regression analyses involving 1-year and 2-year all-cause mortality. All variables tested provided superior accuracy regarding the former than the latter. For both outcomes, BODE was the most accurate predictor, followed by pro-adrenomedullin or BOD, and pro-adrenomedullin was the most discriminative blood biomarker studied.

The combination of each biomarker with BODE improved predictive power significantly compared to using BODE alone (online supplementary tables E1 and E2). The greatest accuracy was achieved with proadrenomedullin plus BODE (C statistic 0.818, $\mathrm{p}<0.001$ for 1 -year mortality; C statistic $0.750, \mathrm{p}<0.001$ for 2 -year mortality). For 1-year mortality, adding pro-adrenomedullin to BODE resulted in a net correct reclassification (95\% CI) of $25.1(2.0-48.2) \%$ of patients $(\mathrm{p}=0.03$; added-value Chi-squared 16.31, $\mathrm{p}<0.0001)$ relative to using BODE alone. For 2-year mortality, there was a net correct reclassification of $12.3(-4.1-28.8) \%$ of patients $(\mathrm{p}=0.14$; added-value Chi-squared $13.65, \mathrm{p}=0.0002)$ for 2 -year mortality. 
TABLE 3 Cox regression model including the BODE components and pro-adrenomedullin to predict 2-year all-cause mortality

Likelihood ratio Chi-squared

\section{Univariate model}

Body mass index

Post-bronchodilator FEV1 \% pred

mMRC dyspnoea score

6-min walk distance

Pro-adrenomedullin

Multivariate model

Body mass index

Post-bronchodilator FEV1 \% pred

mMRC dyspnoea score

6-min walk distance

Pro-adrenomedullin

\section{9}

3.9

27.8

23.5

31.7

8.5

0.3

2.2

7.5

13.0
$\operatorname{HR}(95 \% \mathrm{Cl})$

p-value

For each variable, the hazard ratio (HR) is based on an increment of one-quartile. BODE: body mass, airflow obstruction, dyspnoea, exercise capacity index; FEV1: forced expiratory volume in $1 \mathrm{~s} ; \%$ pred: \% predicted; mMRC: modified Medical Research Council.

Pro-adrenomedullin plus BOD showed superior ability to forecast 1-year or 2-year nonsurvival compared to BODE alone. Adding pro-adrenomedullin to BOD produced a net correct reclassification index (95\% CI) of $41.2(15.6-66.8) \%$ of patients $(\mathrm{p}=0.0016$; added-value Chi-squared 19.13, $\mathrm{p}<0.0001)$ for 1 -year mortality and $8.8(-10.6-28.3) \%$ of patients $(\mathrm{p}=0.37$; added-value Chi-squared $16.22, \mathrm{p}<0.0001)$ for 2 -year mortality, relative to using BOD alone.

Considering all individual BODE components plus pro-adrenomedullin in a multivariable Cox regression model revealed that pro-adrenomedullin, body mass index, and 6MWD, in descending order of strength, contributed to 2 -year survival prediction, but FEV1 \% pred and dyspnoea score did not (table 3). Figure 2 shows dichotomised Kaplan-Meier 2-year survival curves based on optimised cut-offs for BODE (4 out of 10 points) and pro-adrenomedullin; online supplementary figure E1 depicts such curves for the other studied plasma biomarkers. The optimised pro-adrenomedullin cut-off was estimated at $0.75 \mathrm{nmol} \cdot \mathrm{L}^{-1}$, reflecting $68.1 \%$ sensitivity and $70.7 \%$ specificity.

Based on optimised cut-offs for BODE, BOD (4 out of 7 points) and pro-adrenomedullin, we devised two combined mortality prediction indices, "BODE-A" and, for when 6MWD is unobtainable, "BOD-A". Both have three categories, associated with an increasing 2-year mortality rate: low risk (clinical index and biomarker below respective cut-offs), intermediate risk (one of the clinical index or biomarker below cutoffs and the other at/above cut-offs) or high risk (both the clinical index and biomarker at/above cut-offs). These categories and their corresponding 2-year all-cause mortality rates and survival curves are presented in figure 3. To identify 2-year survivors, low-risk BODE-A status had $86.0 \%$ sensitivity and $44.5 \%$
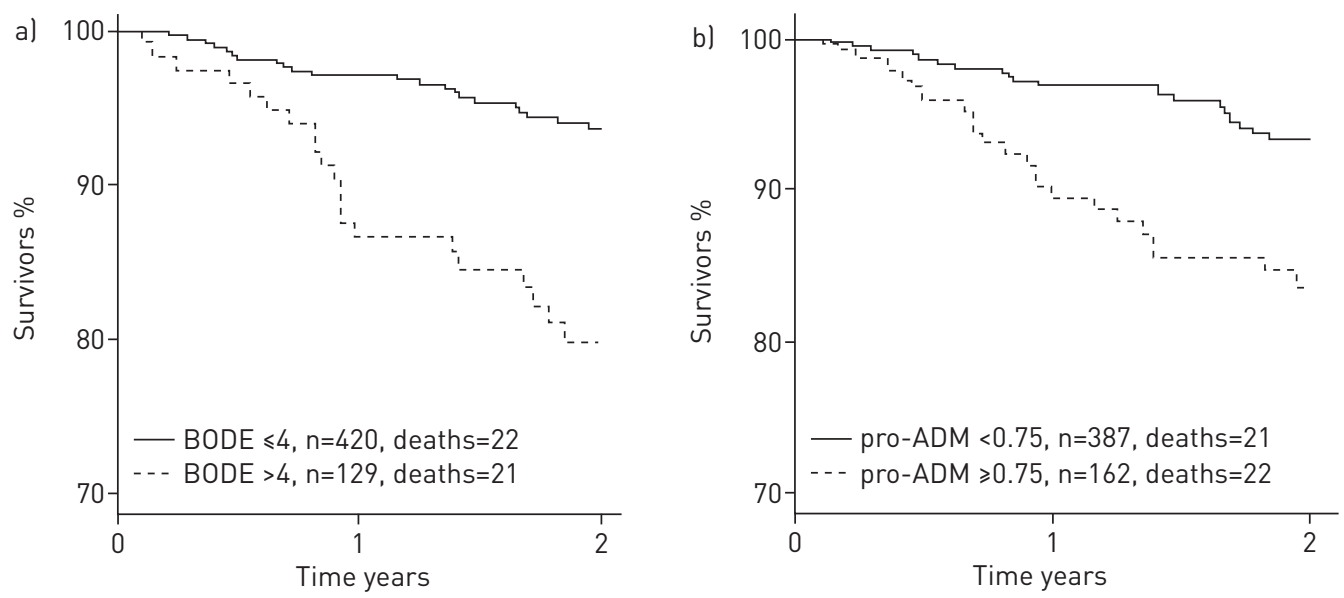

FIGURE 2 Dichotomised 2-year Kaplan-Meier survival curves based on optimised cut-offs for a) body mass, airflow obstruction, dyspnoea, exercise capacity index (BODE) (4 points) and b) pro-adrenomedullin (pro-ADM) $\left(0.75 \mathrm{nmol} \cdot \mathrm{L}^{-1}\right)$. 

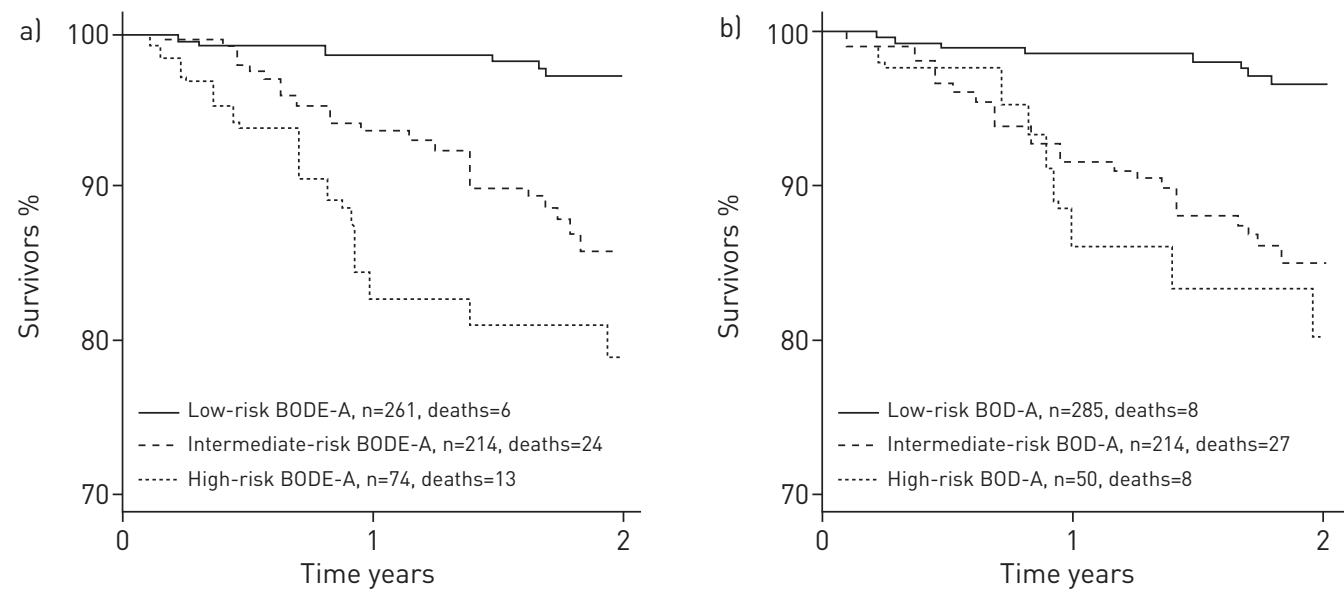

FIGURE 3 Kaplan-Meier 2-year survival curves by risk category according to a) the "BODE-A" index combining BODE (body mass, airflow obstruction, dyspnoea, exercise capacity) and pro-adrenomedullin or b) the "BOD-A" index combining BOD (body mass, airflow obstruction, dyspnoea) and pro-adrenomedullin. These indices, based on the optimised cut-offs for BODE (4 points), BOD (4 points) and pro-adrenomedullin $\left(0.75 \mathrm{nmol} \cdot \mathrm{L}^{-1}\right)$, each had three allcause mortality risk categories: low (both BODE or BOD and pro-adrenomedullin below cut-offs), intermediate (either BODE or BOD and pro-adrenomedullin at or above cut-offs) and high (both BODE and BOD and pro-adrenomedullin at or above cut-offs). The three BOD components were scored according to the same cut-offs as in BODE [2].

specificity; low-risk BOD-A status had $81.4 \%$ sensitivity and $54.7 \%$ specificity. To identify 2-year nonsurvivors, high-risk BODE-A classification had 30.2\% sensitivity and $87.9 \%$ specificity; high-risk BOD-A classification had $18.5 \%$ sensitivity and $91.7 \%$ specificity.

Notably, unavailable 6MWD data was informative in this study: compared to the 549 patients analysed, the 51 excluded for this cause had significantly higher 2 -year mortality $(7.8 \%$ versus $21.6 \%, \mathrm{p}=0.003)$. In post hoc sensitivity analysis using a standard imputation method (HotDeck imputation) BODE accuracy increased. However, combined with BODE, pro-adrenomedullin continued to significantly improve 1-year and 2-year mortality prediction compared to using BODE alone (table 4). A second imputation method, using the worst possible BODE score for $6 \mathrm{MWD}, 3$ points, in these 51 patients, produced similar results (data not shown).

\section{Discussion \\ Interpretation of the findings \\ Pro-adrenomedullin as a mortality predictor}

In this analysis of a well-characterised international cohort from the first multicentre study investigating the biomarker's mortality prediction potential in COPD, pro-adrenomedullin was a powerful prognostic determinant in the stable disease state. Of plasma biomarkers that we studied, pro-adrenomedullin most accurately and significantly foretold 1-year and 2-year all-cause mortality. Moreover, pro-adrenomedullin forecasted these outcomes with similar accuracy as did BODE, and remained significantly associated with 2-year nonsurvival after adjustment for the BODE components. Hence, circulating pro-adrenomedullin itself potentially may be regarded as an independent multidimensional disease assessment tool in COPD.

\section{Pro-adrenomedullin and BODE to predict mortality in COPD}

To our knowledge, PROMISE-COPD was the first multicentre study primarily designed to investigate whether adding a systemic biomarker could enhance BODE prognostic power in COPD. We found that pro-adrenomedullin, used with BODE, indeed significantly improved 1-year and 2-year mortality prediction over that achieved by applying BODE alone. Importantly, for 1-year mortality, improvement occurred in every seventh patient $(p=0.02)$. The incremental discrimination from combining proadrenomedullin with BODE in PROMISE-COPD at least equalled that from adding four circulating biomarkers to established clinical/laboratory risk factors in cardiovascular patients [20].

A much smaller, single-centre study [21] found in multivariable analysis that serum C-reactive protein and BODE independently predicted long-term all-cause mortality. However, the additive value of C-reactive protein in that investigation remains uncertain, as neither the $\mathrm{C}$ statistic, Cox analysis, nor the net reclassification improvement for the biomarker alone or combined with BODE were reported. Moreover, cardiovascular disease, shown to correlate with C-reactive protein levels, was not formally evaluated. 
TABLE 4 Sensitivity analysis: Cox regression models to predict 1-year and 2-year all-cause mortality in patients with stable chronic obstructive pulmonary disease, with missing 6-min walk test data imputed (Hotdeck imputation)

Likelihood ratio Chi-squared

p-value

C statistic

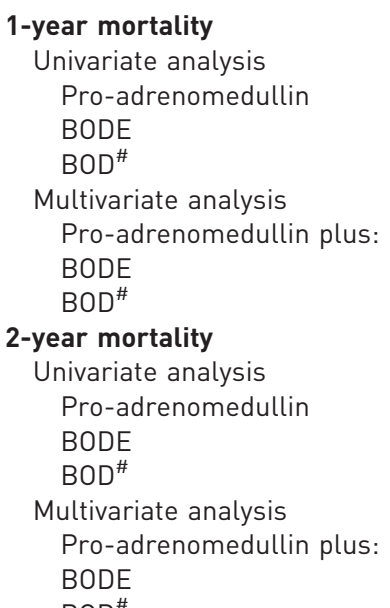

$\begin{array}{ccc}24.38 & <0.001 & 0.715 \\ 33.55 & <0.001 & 0.759 \\ 24.41 & <0.001 & 0.714 \\ & & \\ 53.33^{\circ} & <0.001 & 0.829 \\ 46.82 & <0.001 & 0.815 \\ & & \\ 26.98 & <0.001 & 0.663 \\ 38.03 & <0.001 & 0.711 \\ 30.91 & <0.001 & 0.688 \\ & & \\ 59.46^{+} & & 0.771 \\ 55.60 & <0.001 & 0.764\end{array}$

Analysed cohort $n=594$ (549 with all data available plus the $45(88.2 \%)$ out of 51 patients with missing 6 -min walk distance but available proadrenomedullin data). p-values compare variables with the null hypothesis. BODE: body mass, airflow obstruction, dyspnoea, exercise capacity index. " : BOD comprises BODE without the exercise capacity measurement; the three BOD components are scored according to the same cut-offs as they are in BODE [2], therefore, the "BOD index" ranges, in increasing severity, from 0 to 7. ": for 1-year mortality prediction in this sensitivity analysis, value added of pro-adrenomedullin with BODE Chi-squared 19.78, $p<0.001 .{ }^{+}$: for 2 -year mortality prediction in this sensitivity analysis, value added of pro-adrenomedullin with BODE Chi-squared 21.43, $\mathrm{p}<0.001$.

Additionally, and notably, in the single-centre pro-adrenomedullin study, C-reactive protein was nonsignificant in predicting 2-year mortality after multivariable adjustment including pro-adrenomedullin [14].

Lately, an analysis from a large COPD cohort suggested that adding interleukin-6, or even interleukin-6 plus six other biomarkers to the BODE components and two additional clinical variables significantly improved mortality prediction compared to using the six clinical variables alone [7]. While this approach commendably aims to capture the complexity of a multidimensional disease, determining 13 variables may hamper applicability.

TABLE 5 Pathophysiological mechanisms supporting the role of adrenomedullin as a local and systemic anti-inflammatory factor, produced in response to chronic inflammation, infection or hypoxia in chronic obstructive pulmonary disease

Lipopolysaccharide, hypoxia and shear stress, encountered in conditions related to endothelial damage and infection, are associated with increased adrenomedullin levels

Adrenomedullin immunoreactivity has been reported in bronchial mucosa and glands and type II pneumocytes

Adrenomedullin is expressed by several immune system cells, including macrophages, monocytes and T-cells, in lymphoid organs, including the lung

Adrenomedullin downregulates serum and lung levels of a wide spectrum of inflammatory mediators [32], including:

cytokines (tumour necrosis factor- $\alpha$, interleukin (IL)-6, IL-1 $\beta$, IL-12 and interferon- $\gamma$ )

chemokines (macrophage inflammatory protein-2 and RANTES)

serum amyloid $\mathrm{A}$

nitric oxide

Adrenomedullin upregulates systemic and local levels of the anti-inflammatory cytokine IL-10

Adrenomedullin inhibits neutrophil binding to vascular endothelial cells and subsequent neutrophil emigration in the lung

Adrenomedullin upregulates $\beta$-adrenergic agonist levels, a phenomenon observed in acute asthma and thought to represent a preventive mechanism against bronchoconstriction [31]

Hypoxia leads to adrenomedullin upregulation through the hypoxia inducible factor-1 pathway, which interacts with nuclear factor $\kappa B$ to promote

the expression of inflammatory genes [31, 33-35]. 
Pro-adrenomedullin as an alternative to the 6MWT for mortality prediction

The present analysis demonstrated that in stable COPD, baseline pro-adrenomedullin concentration plus the three "non-6MWD" BODE components more accurately predicted 1-year or 2-year mortality than did the original BODE including the 6MWD. This observation suggests that pro-adrenomedullin measurement may represent an alternative to the 6MWT for mortality prediction. Our multivariable analysis identified $6 \mathrm{MWD}$ as an independent 2-year nonsurvival predictor. Others found 6MWD to be valuable in prognosticating death and other adverse outcomes, besides its use for other important reasons, such as before-and-after treatment comparisons and to measure functional status in COPD [16, 22]. Although 6MWD proved to be independently associated with 2-year all-cause mortality in the multivariate analysis of all BODE components in our study, in contrast to the mMRC and FEV1\% pred, it has been tentatively excluded from the new "BOD-A" index. This apparent discrepancy can be justified only by fact that the $6 \mathrm{MWD}$ is irrefutably the most difficult item to be obtained clinically in the BODE grading system. Accordingly, the 6MWT has downsides posing barriers to BODE adoption $[5,6]$ : the test is relatively complex and time-consuming, and requires a 30-m track, a trained examiner certified in cardiopulmonary resuscitation with a minimum of an American Health Association-approved course in basic life support, and supplies and facilities for rapid medical emergency response. Additionally, the 6MWT may be burdensome for many patients, particularly the elderly, frail or those with musculoskeletal, neuromuscular or peripheral arterial disease $[2,23]$. Indeed, previous attempts, such as the age, dyspnoea and airflow obstruction $(\mathrm{ADO})$ score, have used a similar approach, i.e. refraining from the 6MWD and aiming to increase the applicability of COPD grading systems outside of the specialised respiratory medicine settings [23]. Recently, 6MWD has been shown to lack independent association with computed tomography (CT) density data in an unselected COPD population, thus portending that reduction in the distance walked in the $6 \mathrm{MWT}$ can be attributed, besides to lung parenchymal destruction caused by COPD itself, also to common comorbidities, such as cardiovascular and musculoskeletal disorders [24]. In this context, the fact that patients with musculoskeletal or neuromuscular disorders preventing walking at study inclusion were excluded from the study might have influenced our results. Conversely, the mMRC, which in our study did not independently correlate with mortality, remained an independent predictor only for the CT parameters related to the percentage of lung area with markedly reduced lung density values, i.e. reflecting the extent of emphysematous destructive changes. This confirms that dyspnoea perception is related to alveolar surface reduction, as assessed either by CT parameters or diffusing capacity of the lung for carbon monoxide [24, 25].

Notably, despite a musculoskeletal or neuromuscular disorder preventing walking being a study exclusion criterion, $8.0 \%$ of the PROMISE-COPD cohort had unavailable 6MWD values, by far the commonest grounds for ineligibility for the present analysis. Measuring a blood biomarker rather than 6MWD presumably would offer advantages of speed, simplicity, accessibility and possibly cost. Indeed, in Switzerland, estimated current 6MWT costs approach $€ 40$, while a pro-adrenomedullin determination is estimated to cost $\sim € 15-25$. Importantly, any such advantages need confirmation in a prospective, comparative study.

\section{Pathophysiological basis for pro-adrenomedullin mortality prediction ability}

Our observation that circulating pro-adrenomedullin independently predicts all-cause mortality in COPD patients even after adjusting for the BODE variables suggests that this adrenomedullin surrogate reflects COPD pathophysiological processes and predictive factors uncaptured, or suboptimally captured, by the BODE domains.

Inflammation-induced adrenomedullin expression has been reported in numerous tissues and in vitro models, including vascular endothelial cells, vascular smooth muscle cells, fibroblasts, neurons, macrophages and adipocytes, as well as undifferentiated mesenchymal stem cells and peripheral blood mononuclear cell-derived macrophages [12, 26-30]. Thus, in contrast to CALC I and CALC II products, such as procalcitonin, inflammation-related adrenomedullin production appears to occur ubiquitously in all cell types, independently of the differentiation state [12].

It is tempting, and supported by several observations [31-36] (table 5), to speculate that inflammation, infection and hypoxia upregulate production of adrenomedullin as an endogenous immunomodulatory factor, in an attempt to restore homeostasis in COPD. Interestingly, although adrenomedullin appears to be protective in animal models of infection, we found that elevated pro-adrenomedullin is associated with increased mortality in patients with COPD. Previous experimental work examining adrenomedullin in cardiovascular disease also found that adrenomedullin has beneficial properties [37], whereas clinical studies demonstrated that high levels of pro-adrenomedullin were associated with worse patient outcomes $[38,39]$. Conceptually, this seeming dichotomy probably represents a "fire versus firefighter" epiphenomenon, where the proportional presence of firefighters at a fire reflects their protective, rather than causative, role [40]. Therefore, elevated pro-adrenomedullin levels seen with increased illness severity 
and mortality might represent an endogenous, protective role for adrenomedullin [40], in an attempt to decrease inflammation, apoptosis and infection-induced organ damage [41, 42]. Henceforth, higher proadrenomedullin levels would reflect greater inflammatory stimuli or more pronounced systemic repercussions of the disease, possibly explaining this biomarker's predictive properties in COPD and other respiratory conditions, such as pneumonia, sepsis and dyspnoea with and without heart failure [40, 43-48].

\section{Clinical and research implications of the findings}

What are the possible clinical and research implications of our findings? Pro-adrenomedullin, BODE-A or BOD-A levels might help guide site-of-care decisions in ambiguous cases. In addition, at least theoretically, more accurately identifying COPD patients at higher death risk could facilitate aggressive yet selective early interventions to decrease or avert mortality and other adverse outcomes. Such interventions might include intensified follow-up, e.g. via telemonitoring, pharmacotherapy, noninvasive ventilation or pulmonary rehabilitation. Since patients with severe COPD generally require one to three hospitalisations annually, any effective early intervention potentially may considerably decrease healthcare resource consumption. Such an approach has been valuable in cardiovascular disease and was recently proposed for community-acquired pneumonia [49]. Interventional trials should be conducted of pro-adrenomedullin-aided site-of-care assignment or other clinical decision-making to clarify this biomarker's role in everyday COPD management [50]. Furthermore, application of pro-adrenomedullin, BODE-A, or BOD-A should be considered to identify at-risk patients for recruitment to placebo-controlled clinical trials of modalities to prolong survival in COPD, thereby increasing statistical power, decreasing sample size demands and speeding intervention development. Finally, it could be expected that pro-adrenomedullin levels would be differently upregulated beyond airflow limitation in different COPD phenotypes, i.e. according to the presence and severity of flogistic process or distinctive comorbidities. Within this context, whether proadrenomedullin could be used to differentiate between the two main mechanisms of airflow obstruction in COPD, namely conductive airway inflammation and a parenchymal disruption $[51,52]$, perhaps giving the degree of arterial hypoxaemia, deserves further evaluation.

\section{Strengths and limitations of the study}

This study's strengths and weaknesses merit consideration. Among the former were the investigator-driven, multinational, multicentre, prospective design, and the standardised, comprehensive baseline patient assessment, including exercise capacity and novel blood biomarkers. Study limitations include the smaller cohort size relative to epidemiological studies, noninclusion of never-smokers, absence of standardised baseline lung CT and lack of a long-term, e.g. 5-year, follow-up confirming suggested cut-off values. Lastly, relatively low mortality may have under-powered the analysis to detect weak but statistically significant associations with death. Conversely, the low event rate most probably rendered conservative the findings regarding the significant predictive aptitude of pro-adrenomedullin, i.e. cohorts with higher mortality rates potentially might reveal stronger associations between pro-adrenomedullin and death than those reported here.

\section{Conclusion}

In summary, pro-adrenomedullin appears to be an accurate, significant and independent predictor of longterm all-cause mortality in patients with clinically stable COPD. In this setting, pro-adrenomedullin significantly improves 1-year or 2-year mortality prediction when combined with BODE, or with the three non-6MWT BODE components, compared to prognostication using BODE alone.

\section{Acknowledgements}

The authors' affiliations are as follows. D. Stolz: Dept of Pneumology, University Hospital, Basel, Switzerland; K. Kostikas: Dept of Pneumonology, University Thessaly Medical School, Larissa, Greece; F. Blasi: Dept of Pathophysiology and Transplantation, Università degli Studi di Milano, Milan, Italy; W. Boersma: Dept of Pneumology, Medisch Centrum Alkmaar, Alkmaar, the Netherlands; B. Milenkovic: Faculty of Medicine, University of Belgrade, and Clinic for Pulmonary Diseases, Clinical Centre of Serbia, Belgrade, Serbia; A. Lacoma: Dept of Microbiology, Hospital Universitari Germans Trias i Pujol, Badalona, Spain; R. Louis: Dept of Pneumology, CHU Liege, University of Liege, GIGAI Research Group, Liege, Belgium; J.G. Aerts: Dept of Pneumology, Amphia Hospital/Erasmus MC, Breda, the Netherlands; T. Welte: Dept of Pneumology, Medizinische Hochschule Hannover, Hannover, Germany; A. Torres: Dept of Pneumology, Hospital Clinic - IDIBAPS, Ciber de Enfermedades Respiratorias, Barcelona, Spain; G.G.U. Rohde: Dept of Respiratory Medicine, Maastricht University Medical Center, Maastricht, The Netherlands; L. Boeck: Dept of Pneumology, University Hospital, Basel, Switzerland; Janko Rakic: Dept of Pneumology, University Hospital, Basel, Switzerland; A. Scherr: Dept of Pneumology, University Hospital, Basel, Switzerland; S. Hertel: Clinical Diagnostics Division, Thermo Scientific Biomarkers, Brahms GmbH, Hennigsdorf, Germany; S. Giersdorf: Clinical Diagnostics Division, Thermo Scientific Biomarkers, Brahms GmbH, Hennigsdorf, Germany; M. Tamm: Dept of Pneumology, University Hospital, Basel, Switzerland.

We thank Marilena Pappalettera and Paola Casteliotti (Università degli Studi di Milano, Milan, Italy), Markos Minas (in memoriam) (University Thessaly Medical School, Larissa, Greece), Alicia Marin (Hospital Universitari Germans Trias i Pujol, Badalona, Spain) and particularly Anja Meyer (University Hospital Basel, Basel, Switzerland) for the exceptional 
support in data management and/or patient inclusion and follow-up. Robert J. Marlowe (Spencer-Fontayne Corporation, Jersey City, NJ, USA) edited the manuscript.

\section{References}

1 Global Initative for Chronic Obstructive Lung Disease. Global Strategy for Diagnosis, Management and Prevention of Chronic Obstructive Pulmonary Disease. Global Initiative for Chronic Obstructive Lung Disease, 2011.

2 Celli BR, Cote CG, Marin JM, et al. The body-mass index, airflow obstruction, dyspnea, and exercise capacity index in chronic obstructive pulmonary disease. N Engl J Med 2004; 350: 1005-1012.

3 Marin JM, Alfageme I, Almagro P, et al. Multicomponent indices to predict survival in COPD: the COCOMICS study. Eur Respir J 2013; 42: 323-332.

4 Divo M, Cote C, de Torres JP, et al. Comorbidities and risk of mortality in patients with chronic obstructive pulmonary disease. Am J Respir Crit Care Med 2012; 186: 155-161.

5 Jones RC, Donaldson GC, Chavannes NH, et al. Derivation and validation of a composite index of severity in chronic obstructive pulmonary disease: the DOSE Index. Am J Respir Crit Care Med 2009; 180: 1189-1195.

6 Briggs AH, Glick HA, Lozano-Ortega G, et al. Is treatment with ICS and LABA cost-effective for COPD? Multinational economic analysis of the TORCH study. Eur Respir J 2010; 35: 532-539.

7 Celli BR, Locantore N, Yates J, et al. Inflammatory biomarkers improve clinical prediction of mortality in chronic obstructive pulmonary disease. Am J Respir Crit Care Med 2012; 185: 1065-1072.

8 Koutsokera A, Stolz D, Loukides S, et al. Systemic biomarkers in exacerbations of COPD: the evolving clinical challenge. Chest 2012; 141: 396-405.

9 Kostikas K, Bakakos P, Papiris S, et al. Systemic biomarkers in the evaluation and management of COPD patients: are we getting closer to clinical application? Curr Drug Targets 2013; 14: 177-191.

10 Struck J, Tao C, Morgenthaler NG, et al. Identification of an adrenomedullin precursor fragment in plasma of sepsis patients. Peptides 2004; 25: 1369-1372.

11 Morgenthaler NG, Struck J, Alonso C, et al. Measurement of midregional proadrenomedullin in plasma with an immunoluminometric assay. Clin Chem 2005; 51: 1823-1829.

12 Linscheid P, Seboek D, Zulewski $\mathrm{H}$, et al. Autocrine/paracrine role of inflammation-mediated calcitonin generelated peptide and adrenomedullin expression in human adipose tissue. Endocrinology 2005; 146: 2699-2708.

13 Vizza CD, Letizia C, Sciomer S, et al. Increased plasma levels of adrenomedullin, a vasoactive peptide, in patients with end-stage pulmonary disease. Regul Pept 2005; 124: 187-193.

14 Stolz D, Christ-Crain M, Morgenthaler NG, et al. Plasma pro-adrenomedullin but not plasma pro-endothelin predicts survival in exacerbations of COPD. Chest 2008; 134: 263-272.

15 Brusasco V, Crapo R, Viegi G, et al. Coming together: the ATS/ERS consensus on clinical pulmonary function testing. Eur Respir J 2005; 26: 1-2.

16 ATS Committee on Proficiency Standards for Clinical Pulmonary Function Laboratories. ATS statement: guidelines for the six-minute walk test. Am J Respir Crit Care Med 2002; 166: 111-117.

17 Smith JG, Newton-Cheh C, Hedblad B, et al. Distribution and correlates of midregional proadrenomedullin in the general population. Clin Chem 2009; 55: 1593-1595.

18 Harrell FEJ. Regression Modeling Strategies: with Application to Linear Models, Logistic Regression, and Survival Analysis. New York, Springer, 2001.

19 Pencina MJ, D’Agostino RB Sr, D’Agostino RB Jr, et al. Evaluating the added predictive ability of a new marker: from area under the ROC curve to reclassification and beyond. Stat Med 2008; 27: 157-172.

20 Zethelius B, Berglund L, Sundström J, et al. Use of multiple biomarkers to improve the prediction of death from cardiovascular causes. N Engl J Med 2008; 358: 2107-2116.

21 Liu SF, Wang CC, Chin CH, et al. High value of combined serum C-reactive protein and BODE score for mortality prediction in patients with stable COPD. Arch Bronconeumol 2011; 47: 427-432.

22 Spruit MA, Polkey MI, Celli B, et al. Predicting outcomes from 6-minute walk distance in chronic obstructive pulmonary disease. J Am Med Dir Assoc 2012; 13: 291-297.

23 Puhan MA, Garcia-Aymerich J, Frey M, et al. Expansion of the prognostic assessment of patients with chronic obstructive pulmonary disease: the updated BODE index and the ADO index. Lancet 2009; 374: 704-711.

24 Camiciottoli G, Bigazzi F, Bartolucci M, et al. BODE-index, modified BODE-index and ADO-score in chronic obstructive pulmonary disease: relationship with COPD phenotypes and CT lung density changes. COPD 2012; 9: 297-304.

25 Camiciottoli G, Bartolucci M, Maluccio NM, et al. Spirometrically gated high-resolution CT findings in COPD: lung attenuation versus lung function and dyspnea severity. Chest 2006; 129: 558-564.

26 Beltowski J, Jamroz A. Adrenomedullin - what do we know 10 years since its discovery? Pol J Pharmacol 2004; 56: $5-27$.

27 Kubo A, Minamino N, Isumi Y, et al. Production of adrenomedullin in macrophage cell line and peritoneal macrophage. J Biol Chem 1998; 273: 16730-16738.

28 Li Y, Totsune K, Takeda K, et al. Differential expression of adrenomedullin and resistin in 3T3-L1 adipocytes treated with tumor necrosis factor-alpha. Eur J Endocrinol 2003; 149: 231-238.

29 Cameron VA, Fleming AM. Novel sites of adrenomedullin gene expression in mouse and rat tissues. Endocrinology 1998; 139: 2253-2264.

30 Fukai N, Yoshimoto T, Sugiyama T, et al. Concomitant expression of adrenomedullin and its receptor components in rat adipose tissues. Am J Physiol Endocrinol Metab 2005; 288: E56-E62.

31 Kohno M, Hanehira T, Hirata K, et al. An accelerated increase of plasma adrenomedullin in acute asthma. Metabolism 1996; 45: 1323-1325.

32 Gonzalez-Rey E, Chorny A, Varela N, et al. Urocortin and adrenomedullin prevent lethal endotoxemia by downregulating the inflammatory response. Am J Pathol 2006; 168: 1921-1930.

33 Pfeil U, Aslam M, Paddenberg R, et al. Intermedin/adrenomedullin-2 is a hypoxia-induced endothelial peptide that stabilizes pulmonary microvascular permeability. Am J Physiol Lung Cell Mol Physiol 2009; 297: L837-L845.

34 Marinoni E, Pacioni K, Sambuchini A, et al. Regulation by hypoxia of adrenomedullin output and expression in human trophoblast cells. Eur J Obstet Gynecol Reprod Biol 2011; 154: 146-150. 
MacManus CF, Campbell EL, Keely S, et al. Anti-inflammatory actions of adrenomedullin through fine tuning of HIF stabilization. FASEB J 2011; 25: 1856-1864.

36 Fujikura T, Okubo K. Adrenomedullin level in the nasal discharge from allergic rhinitis cohort. Peptides 2011; 32: 368-373.

37 Eto T, Kitamura K, Kato J. Biological and clinical roles of adrenomedullin in circulation control and cardiovascular diseases. Clin Exp Pharmacol Physiol 1999; 26: 371-380.

38 Khan SQ, O'Brien RJ, Struck J, et al. Prognostic value of midregional pro-adrenomedullin in patients with acute myocardial infarction: the LAMP (Leicester Acute Myocardial Infarction Peptide) study. J Am Coll Cardiol 2007; 49: 1525-1532.

39 Gegenhuber A, Struck J, Dieplinger B, et al. Comparative evaluation of B-type natriuretic peptide, mid-regional pro-A-type natriuretic peptide, mid-regional pro-adrenomedullin, and Copeptin to predict 1-year mortality in patients with acute destabilized heart failure. J Card Fail 2007; 13: 42-49.

40 Huang DT, Angus DC, Kellum JA, et al. Midregional proadrenomedullin as a prognostic tool in communityacquired pneumonia. Chest 2009; 136: 823-831.

41 Shindo T, Kurihara H, Maemura K, et al. Hypotension and resistance to lipopolysaccharide-induced shock in transgenic mice overexpressing adrenomedullin in their vasculature. Circulation 2000; 101: 2309-2316.

42 Itoh T, Obata H, Murakami S, et al. Adrenomedullin ameliorates lipopolysaccharide-induced acute lung injury in rats. Am J Physiol Lung Cell Mol Physiol 2007; 293: L446-L452.

43 Christ-Crain M, Morgenthaler NG, Stolz D, et al. Pro-adrenomedullin to predict severity and outcome in community-acquired pneumonia [ISRCTN04176397]. Crit Care 2006; 10: R96.

44 Krüger S, Ewig S, Giersdorf S, et al. Cardiovascular and inflammatory biomarkers to predict short- and long-term survival in community-acquired pneumonia: results from the German Competence Network, CAPNETZ. Am J Respir Crit Care Med 2010; 182: 1426-1434.

45 Albrich WC, Dusemund F, Rüegger K, et al. Enhancement of CURB65 score with proadrenomedullin (CURB65-A) for outcome prediction in lower respiratory tract infections: derivation of a clinical algorithm. BMC Infect Dis 2011; 11: 112

46 Bello S, Lasierra AB, Mincholé E, et al. Prognostic power of proadrenomedullin in community-acquired pneumonia is independent of aetiology. Eur Respir J 2012; 39: 1144-1155.

47 Maisel A, Mueller C, Nowak RM, et al. Midregion prohormone adrenomedullin and prognosis in patients presenting with acute dyspnea: results from the BACH (Biomarkers in Acute Heart Failure) trial. J Am Coll Cardiol 2011; 58: 1057-1067.

48 Schuetz P, Christ-Crain M, Morgenthaler NG, et al. Circulating precursor levels of endothelin-1 and adrenomedullin, two endothelium-derived, counteracting substances, in sepsis. Endothelium 2007; 14: 345-351.

49 Ewig S, Torres A. Community-acquired pneumonia as an emergency: time for an aggressive intervention to lower mortality. Eur Respir J 2011; 38: 253-260.

50 Bakke PS, Rönmark E, Eagan T, et al. Recommendations for epidemiological studies on COPD. Eur Respir J 2011; 38: 1261-1277.

51 Han MK, Kazerooni EA, Lynch DA, et al. Chronic obstructive pulmonary disease exacerbations in the COPDGene study: associated radiologic phenotypes. Radiology 2011; 261: 274-282.

52 Camiciottoli G, Bigazzi F, Paoletti M, et al. Pulmonary function and sputum characteristics predict computed tomography phenotype and severity of COPD. Eur Respir J 2013; 42: 626-635. 